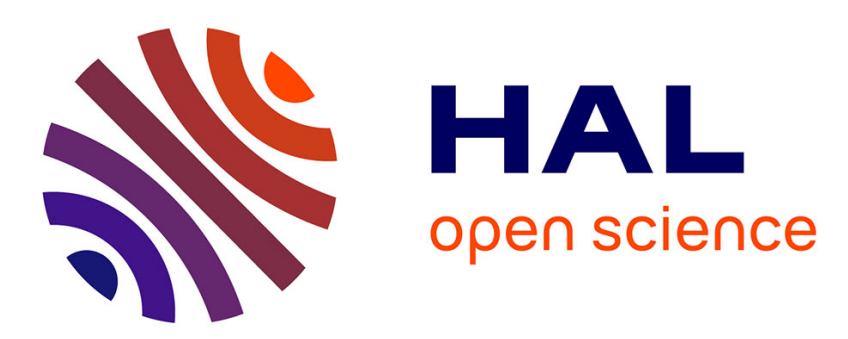

\title{
Discussion of "Approximate Analytical Solutions for the Colebrook Equation" by Ali R. Vatankhah
}

Dejan Brkić, Pavel Praks

\section{To cite this version:}

Dejan Brkić, Pavel Praks. Discussion of "Approximate Analytical Solutions for the Colebrook Equation" by Ali R. Vatankhah. Journal of Hydraulic Engineering, 2020, 146 (2), pp.07019011. 10.1061/(asce)hy.1943-7900.0001667 . hal-02438103

\section{HAL Id: hal-02438103 https://hal.science/hal-02438103}

Submitted on 14 Jan 2020

HAL is a multi-disciplinary open access archive for the deposit and dissemination of scientific research documents, whether they are published or not. The documents may come from teaching and research institutions in France or abroad, or from public or private research centers.
L'archive ouverte pluridisciplinaire HAL, est destinée au dépôt et à la diffusion de documents scientifiques de niveau recherche, publiés ou non, émanant des établissements d'enseignement et de recherche français ou étrangers, des laboratoires publics ou privés. 


\section{Discussion of "Approximate Analytical Solutions for the Colebrook Equation” by Ali R.}

2 Vatankhah, May 2018, Vol. 144, Issue 5, 06018007, Doi: 10.1061/(ASCE)HY.1943-7900.0001454

3

4 Dejan Brkić, PhD, Researcher, IT4Innovations, VŠB - Technical University of Ostrava, 17. listopadu 5 2172/15, 70800 Ostrava, Czech Republic and Research and Development Center "Alfatec", Bulevar

6 Nikole Tesle, 18000 Niš, Serbia, dejanbrkic0611@gmail.com - ORCID id: 0000-0002-2502-0601

7 Pavel Praks, PhD, Researcher, IT4Innovations, VŠB - Technical University of Ostrava, 17. listopadu

8 2172/15, 70800 Ostrava, Czech Republic, pavel.praks@ vsb.cz - ORCID id: 0000-0002-3913-7800

9

10

11

12

$13 \quad \frac{1}{\sqrt{f}}=-2 \cdot \log _{10}\left(\frac{2.51}{R} \cdot \frac{1}{\sqrt{f}}+\frac{\varepsilon}{3.71 \cdot D}\right)$

14 Where $\mathrm{f}$ is Darcy flow friction factor, $\mathrm{R}$ is the Reynolds number, and $\varepsilon / \mathrm{D}$ is the relative roughness of

\section{Approximations based on genetic programming}

The author of the discussed paper (Vatankhah and Kouchakzadeh 2008; 2009, Vatankhah 2014) has made significant contributions related to the Colebrook equation for flow friction (Colebrook 1939; Colebrook and White 1937); Eq. (1): inner pipe surface (all three quantities are dimensionless).

It is underlined that it is important to develop not only accurate (Gregory and Fogarasi 1985, Brkić 2011a; 2012, Winning and Coole 2013) but also computational efficient approximations to the Colebrook equation (Clamond 2009, Giustolisi et al. 2011, Ćojbašić and Brkić 2013, Winning and Coole 2013, Brkić and Ćojbašić 2017). To be computational efficient the approximations need to contain the least possible number of logarithmic and non-integer power expressions (Brkić and Praks 2019). Knowing that appropriately trained artificial neural networks (Özger and Yıldırım 2009, Brkić and Ćojbašić 2016) can simulate the Colebrook equation accurately not knowing the structure of the equation but only knowing the input parameters which are the Reynolds number, $\mathrm{R}$ and the relative roughness, $\varepsilon / \mathrm{D}$ and the 
corresponding output parameter which is the flow friction factor, $f ;\{R, \varepsilon / D\} \rightarrow f$, we tried to use that fact

27 to extract the accurate and computational accurate explicit approximations using artificial intelligent

28 techniques (Smidt and Lipson 2009, Dubčáková 2011), Eq. (2). We used Eureqa, artificial intelligence

29 software tool based on genetic programming (Praks and Brkić 2018a). The symbolic regression approach

30 adopted herein is based upon genetic programming wherein a population of functions is allowed to breed

31 and mutate with the genetic propagation into subsequent generations based upon a survival-of-the-fittest

32 criteria.

$33 \frac{1}{\sqrt{f_{0}}} \approx 1.15 \cdot \alpha+0.569 \cdot \beta+0.292 \cdot \alpha \cdot \beta+0.478 \cdot \sin (0.939 \cdot \alpha-\beta)+0.122 \cdot \sin ^{2}(0.939 \cdot \alpha-\beta)-$

$34 \quad 1.284-0.12 \cdot \alpha^{2}-0.162 \cdot \beta^{2}$

35 Where $\alpha=\log _{10}(\mathrm{R}) ; \beta=-\log _{10}(\varepsilon / \mathrm{D})$.

37 It is revealed that the input parameters the Reynolds number, $\mathrm{R}$ and the relative roughness $\varepsilon / \mathrm{D}$ need to be 38 normalized because their raw form where $\mathrm{R}>1000$ and $\varepsilon / \mathrm{D}<<1$ can cause a problem for the genetic 39 programming tool to recognize the pattern and to estimate flow friction $\mathrm{f}$ accurately and we use $40 \alpha=\log _{10}(\mathrm{R})$ and $\beta=-\log _{10}(\varepsilon / \mathrm{D})$. The practical domain of the Reynolds number, $\mathrm{R}$ is between around 4000 to $41 \quad 10^{8}$ which means it is expressed in relatively large numbers, while the relative roughness, $\varepsilon / \mathrm{D}$ is up to 420.05 which means that it is expressed in relatively small numbers. As explained, the different scale is a 43 problem for the artificial intelligence to recognize pattern (Özger and Yıldırım 2009, Brkić and Ćojbašić

44 2016) where we discovered that the normalization in the form $\alpha=\log _{10}(\mathrm{R})$ and $\beta=-\log _{10}(\varepsilon / \mathrm{D})$ can 45 overwhelm the problem. This means that Eq. (2) practically contain only two logarithmic expressions 46 used only for normalization $\left\{\alpha=\log _{10}(\mathrm{R}) ; \beta=-\log _{10}(\varepsilon / \mathrm{D})\right\} \rightarrow \mathrm{f}$, and not a single non-integer power 47 expression. In that way the estimated relative error of $f_{0}$ calculated through Eq. (2) compared with the 48 accurate friction factor $\mathrm{f} ;\left(\left|\mathrm{f}-\mathrm{f}_{\mathrm{o}}\right| / \mathrm{f}\right) \cdot 100 \%$ is less than $2 \%$. On the other hand the approximation presented 49 with Eq. (2) contains sinus trigonometric function which can introduce higher computational cost. 
50 Introducing one additional logarithmic form, i.e. one more fixed-point iterative step; Eq. (3); Brkić

51 (2017a), the error in this case can be reduced ten times, and it is up to $0.2 \%$. The strategy with

52 acceleration using one additional internal iterative step; two in total including initial starting iteration

53 (Chen 1979, Shorle et al. 1980, Brkić 2011b), is widely used.

$54 \frac{1}{\sqrt{f_{i+1}}} \approx-2 \cdot \log _{10}\left(\frac{2.51}{R} \cdot \frac{1}{\sqrt{f_{i}}}+\frac{\varepsilon}{3.71 \cdot D}\right)$

56 On the other hand, using the raw parameters without normalization; $\{\mathrm{R}, \varepsilon / \mathrm{D}\} \rightarrow \mathrm{f}$ and with acceleration

57 through Eq. (3) the maximal error of no more than $1.55 \%$ is introduced using only one logarithmic form;

58 Eq. (4):

$59 \frac{1}{\sqrt{f_{1}}} \approx-2 \cdot \log _{10}\left(\frac{2.51}{R} \cdot\left(5.05-30.73 \cdot \frac{\varepsilon}{\mathrm{D}}+\frac{3.4 \cdot R+\frac{R^{2}}{469647.7}}{46137.9+\mathrm{R}+\frac{\mathrm{R}^{2}}{3250657.6}+\frac{\frac{\varepsilon}{\mathrm{D}} \cdot \mathrm{R}^{2}}{515.25}}\right)+\frac{\varepsilon}{3.71 \cdot D}\right)$

61 The accelerative procedure through Eq. (3) is very fast where for Eq. (4) the relative error decreases as

62 follow: $\mathrm{f}_{0} \rightarrow 42.7 \%, \mathrm{f}_{1} \rightarrow 1.55 \%, \mathrm{f}_{2} \rightarrow 0.22 \%, \mathrm{f}_{3} \rightarrow 0.036 \%$, etc. The expression for $\mathrm{f}_{0}$ is polynomial and

63 hence very simple, but with the relative error that can reach up to $42.7 \%$ not sufficiently accurate. On the

64 other hand, with two logarithmic expressions, the relative error $f_{2}$ introduced with Eq. (4) is about $0.22 \%$

65 which is almost the same as for $\mathrm{f}_{1}$ introduced with Eq. (2) that requires three logarithmic expressions in total (two for normalization and one for acceleration).

67

\section{Three-point methods}

69 Approximations with virtually three iterative steps are also available (Zigrang and Sylvester 1982,

70 Serghides 1984). Using only function evaluations and one evaluation of the first derivative (Sharma and

71 Arora 2016), the Colebrook equation can be solved practically with the neglected error within one

72 iteration step; Eq. (5), (Džunić et al. 2011). Through the three-point iterative procedure the friction factor

73 is evaluated in points $\mathrm{x}_{0}, \mathrm{y}_{0}$ and $\mathrm{z}_{0}$ where the optimal starting point $\mathrm{x}_{0}$ for the whole domain of the 
74 Colebrook equation is $\mathrm{x}_{0}=7.273124147$ which is chosen after numerous tests where $\mathrm{x}_{0}=\frac{1}{\sqrt{f_{0}}}$. The final

75 solution is at the point $\mathrm{x}_{1}$; Eq. (5) where $\mathrm{f}_{1}=\frac{1}{\sqrt{x_{1}}}$; (Praks and Brkić 2018bc):

76

$$
\left.\begin{array}{c}
\frac{1}{\sqrt{f_{0}}}=\mathrm{x}_{0}=7.273124147 \\
F^{\prime}\left(x_{0}\right)=\frac{\left.1 x_{0}\right)=x_{0}+2 \cdot \log _{10}\left(\frac{2.51 \cdot x_{0}}{R}+\frac{\varepsilon}{3.71 \cdot D}\right)}{\ln (106242) \cdot\left(93121 \cdot x_{0}+10000 \cdot \frac{\varepsilon}{D} \cdot R\right)}+1=\frac{251}{50 \cdot \mathrm{R} \cdot \ln (10) \cdot\left(\frac{100}{371} \cdot \frac{\varepsilon}{\mathrm{D}}+\frac{2.51 \cdot \mathrm{x}_{0}}{\mathrm{R}}\right)}+1 \\
y_{0}=x_{0}-\frac{F\left(x_{0}\right)}{F^{\prime}\left(x_{0}\right)} \\
F\left(y_{0}\right)=y_{0}+2 \cdot \log _{10}\left(\frac{2.51 \cdot y_{0}}{R}+\frac{\varepsilon}{3.71 \cdot D}\right) \\
z_{0}=y_{0}-\frac{F\left(x_{0}\right)}{F\left(x_{0}\right)-2 \cdot F\left(y_{0}\right)} \cdot \frac{F\left(y_{0}\right)}{F^{\prime}\left(x_{0}\right)} \\
F\left(z_{0}\right)=z_{0}+2 \cdot \log _{10}\left(\frac{2.51 \cdot z_{0}}{R}+\frac{\varepsilon}{3.71 \cdot D}\right) \\
x_{1}=z_{0}-\frac{F\left(z_{0}\right)}{F^{\prime}\left(x_{0}\right) \cdot\left[1-2 \cdot \frac{F\left(y_{0}\right)}{F\left(x_{0}\right)}-\left(\frac{F\left(y_{0}\right)}{F\left(x_{0}\right)}\right)^{2}\right] \cdot\left[1-\frac{F\left(z_{0}\right)}{F\left(y_{0}\right)}\right] \cdot\left[1-2 \cdot \frac{F\left(z_{0}\right)}{F\left(x_{0}\right)}\right]} \\
x_{1}=\frac{1}{\sqrt{f_{1}}} \rightarrow f
\end{array}\right\}
$$

77

78 In Eq. (5) is used the first derivative $\mathrm{F}^{\prime}\left(\mathrm{x}_{0}\right)$ of the Colebrook function in point $\mathrm{x}_{0}$ in respect to parameter $\mathrm{x}$,

79 where the Colebrook equation is expressed in the suitable form to start the Newton-Raphson iterative

80 procedure; Eq. (6):

81

$F=\frac{1}{\sqrt{f}}+2 \cdot \log _{10}\left(\frac{2.51}{R} \cdot \frac{1}{\sqrt{f}}+\frac{\varepsilon}{3.71 \cdot D}\right)=0$

82

83 The corresponding VPA (Variable precision arithmetic) approximations of the first derivative F'(x) with

8416 digits precision expression is $=2.180158299154324 /(\mathrm{R} \cdot(0.2695417789757412 \cdot \varepsilon / \mathrm{D}+(2.51 \cdot \mathrm{x}) / \mathrm{R}))+1.0=$

85 $80883.87289862543 /(93121.0 \cdot \mathrm{x}+10000.0 \cdot \mathrm{R} \cdot \varepsilon / \mathrm{D})+1.0$ where $\mathrm{x}=\frac{1}{\sqrt{f}}$

86

\section{Optimization problem}

88 As noted in the discussed paper, the first derivative of the Colebrook equation expressed in the form 89 suitable for the Newton-Raphson procedure in respect to the Reynolds number, $\mathrm{R}$ is used for the 
optimization problem in pipe network simulations (Brkić 2016). For the purpose of this discussion we

91 generated in MATLAB the appropriate first derivative F'(R); Eq. (7):

$92 \quad F^{\prime}(R)=-\frac{251 \cdot \frac{1}{\sqrt{f}}}{50 \cdot R^{2} \cdot \ln (10) \cdot\left(\frac{100}{371} \cdot \frac{\varepsilon}{D}+\frac{2.51}{R} \cdot \frac{1}{\sqrt{f}}\right)}=-\frac{186242 \cdot \frac{1}{\sqrt{f}}}{R \cdot \ln (10) \cdot\left(93121 \cdot \frac{1}{\sqrt{f}}+10000 \cdot R \cdot \frac{\varepsilon}{D}\right)}$

94 The corresponding VPA (Variable precision arithmetic) approximations of the first derivative F'(R) with

9516 digits precision is $=-(186242.0 \cdot x) /\left(23025.85092994046 \cdot \mathrm{R}^{2} \cdot \varepsilon / \mathrm{D}+214419.0264446985 \cdot \mathrm{x} \cdot \mathrm{R}\right)=$

$96-(80883.87289862543 \cdot \mathrm{x}) /(\mathrm{R} \cdot(93121.0 \cdot \mathrm{x}+10000.0 \cdot \mathrm{R} \cdot \varepsilon / \mathrm{D}))$ where $\mathrm{x}=\frac{1}{\sqrt{f}}$

97

\section{Padé polynomials}

99 In the discussed paper, the improved versions of the approximations proposed by Sonnad and Goudar 100 (2006), Mikata and Walczak (2016), Brkić (2017b), Vatankhah and Kouchakzadeh (2008, 2009), and 101 Vatankhah (2014) are shown. In addition, the second logarithmic form in Eq. (12) of the discussed paper 102 can be simplified using Padé approximation (Baker and Graves-Morris 1996$) ; \ln (1-\mathrm{a} \cdot \delta / \mathrm{d})=\ln (1-\theta) \approx \mathrm{p} / \mathrm{q}$ 103 where $\mathrm{p}$ and $\mathrm{q}$ are defined by Eq. (8):

For the domain of applicability of the Colebrook equation that is $0<\varepsilon / \mathrm{D}<0.1$ and $4000<\mathrm{R}<10^{8} ; \theta=\mathrm{a} \cdot \delta / \mathrm{d}$ is

107 always between 0 and $0.22 ; 0<\theta<0.22$, the relative percentage error of the Padé approximation p/q compared with the accurate $\ln (1-\mathrm{a} \cdot \delta / \mathrm{d})$ is between $3.5 \cdot 10^{-8} \%$ and $2 \cdot 10^{-5} \%$. In this case the Padé approximation is very accurate, and also computationally cheap compared with logarithmic function: it is a division of two polynomials of degree of 3; we can call Padé approximation (3/3) because of two

111 polynomials of degree of 3 used (Praks and Brkić 2018d).

112 Further to start Newton-Raphson procedure $\delta_{\mathrm{i}+1}=\delta_{\mathrm{i}}-\zeta\left(\delta_{\mathrm{i}}\right) / \zeta^{\prime}\left(\delta_{\mathrm{i}}\right)$ starting from $\mathrm{i}=0$, in order to evaluate $\delta$, Eq. 113 (12) of the discussed paper need to be rearranged where $\zeta(\delta)=0$, and where $\zeta$ is functional symbol; Eq. (9): 
$114 \zeta(\delta)=\ln (d)+\ln \left(1-\frac{a \cdot \delta}{d}\right)-\delta \approx \ln (d)+\frac{\theta \cdot\left(-0.18333 \cdot \theta^{2}+\theta-1\right)}{-0.05 \cdot \theta^{3}+0.6 \cdot \theta^{2}-1.5 \cdot \theta+1}-\delta=0$

$115 \delta$ is the unknown parameter to be solved, a is constant $\mathrm{a}=2 / \ln (10)=0.8686$ and $\mathrm{d}$ is parameter $\mathrm{d}=\mathrm{c} / \mathrm{b}$ $116 \mathrm{a} \cdot \ln (\mathrm{b})$; where $\mathrm{b}=2.51 / \mathrm{R}$ and $\mathrm{c}=(\varepsilon / \mathrm{D}) / 3.71$.

118 Also, the Newton-Raphson procedure requires the first derivative $\zeta^{\prime}(\delta)=-\mathrm{a} /(\mathrm{d}-\mathrm{a} \cdot \delta)-1$, which is

119 computationally cheap. Because it is equally cheap but slightly more accurate, we use the first derivative of $\zeta(\delta)=\ln (\mathrm{d})+\ln (1-\mathrm{a} \cdot \delta / \mathrm{d})-\delta=0$ and not the derivative of its Padé version. Knowing that $\delta$ ranges between

121 1.61and 13.91, the starting point $\delta_{0}$ for the Newton-Raphson procedure should be selected from that 122 domain; $\delta_{\mathrm{i}+1}=\delta_{\mathrm{i}}-\zeta\left(\delta_{\mathrm{i}}\right) / \zeta^{\prime}\left(\delta_{\mathrm{i}}\right)$ starting from $\mathrm{i}=0$. The Newton-Raphson is faster than the simple fixed-point 123 iterative procedure (Brkić 2017a), so hopefully Eq. (9) should be evaluated only few times; where the 124 stopping criteria is $\delta_{\mathrm{i}+1}-\delta_{\mathrm{i}} \approx 0$.

Disclaimer: The views expressed are those of the authors and may not in any circumstances be regarded 127 as stating an official position of the Alfatec or of the IT4Innovations, VŠB - Technical University 128 Ostrava. Both authors contributed equally to this discussion.

Acknowledgments:

131 This work has been partially funded by the Technology Agency of the Czech Republic, partially by the 132 National Centre for Energy [TN01000007] and partially by the project "Energy System for Grids" 133 [TK02030039]. Work of D.B. has been also supported of the Ministry of Education, Youth, and Sports of 134 the Czech Republic through the National Programme of Sustainability (NPS II) project "IT4Innovations 135 excellence in science" [LQ1602] and by the Ministry of Education, Science, and Technological 136 Development of the Republic of Serbia through the project "Development of new information and 137 communication technologies, based on advanced mathematical methods, with applications in medicine, telecommunications, power systems, protection of national heritage and education” [iii44006]. 
140 Notation

141 The following symbols are used in this discussion:

$142 \mathrm{a}=$ constant, $\mathrm{a}=2 / \ln (10)=0.8686$;

$143 \mathrm{~b}=$ parameter related to Reynolds number (dimensionless);

$144 \mathrm{c}=$ parameter related to $\varepsilon$ and $\mathrm{D}$ (dimensionless);

$145 \mathrm{D}=$ inner diameter of pipe (length; the same unit as for D);

$146 \mathrm{~d}=$ parameter related to $\mathrm{a}, \mathrm{b}$ and $\mathrm{c}$ (dimensionless);

$147 \mathrm{f}=$ Darcy friction factor implicitly related to $\mathrm{R}, \varepsilon$ and $\mathrm{D}$ (dimensionless);

$148 \mathrm{R}=$ Reynolds number (dimensionless);

$149 \mathrm{p}$ and $\mathrm{q}=$ Padé polynomials related to $\theta$;

$150 \mathrm{x}=$ parameter related to friction factor $\mathrm{f}$ (dimensionless);

$151 \mathrm{y}=$ parameter related to $\mathrm{x}$ (dimensionless);

$152 \mathrm{z}=$ parameter related to $\mathrm{y}$ (dimensionless);

$153 \alpha=$ parameter related to the Reynolds number R (dimensionless);

$154 \beta=$ parameter related to $\varepsilon$ and $\mathrm{D}$ (dimensionless);

$155 \delta=$ unknown parameter implicitly related to a and d;

$156 \varepsilon=$ average height of protrusion of inner pipe surface (length; the same unit as for D);

$157 \theta=$ parameter related to $\mathrm{a}, \delta$ and $\mathrm{d}$

158 functional symbols:

$159 \mathrm{~F}-$ functional symbol related to the Colebrook equation

160 ln - Napierian natural logarithm

$161 \log -$ Briggsian logarithm

$162 \sin -$ sine trigonometric function

163 ' - represents first derivative of function

$164 \zeta$ - functional symbol related to parameter $\delta$ 
$166 \mathrm{i}-$ counter

$1670-$ refers to first iteration

$1681-$ refers to second iteration

\section{References:}

171 Baker, G. A., and Graves-Morris, P. (1996). Padé approximants. Encyclopedia of Mathematics and its

172 Applications, Cambridge University Press. https://doi.org/10.1017/CBO9780511530074

173 Brkić, D. (2011a). "Review of explicit approximations to the Colebrook relation for flow friction."

174 Journal of Petroleum Science and Engineering, 77(1), 34-48. https://doi.org/10.1016/j.petrol.2011.02.006

175 Brkić, D. (2011b). "New explicit correlations for turbulent flow friction factor." Nuclear Engineering and 176 Design, 241(9), 4055-4059. https://doi.org/10.1016/j.nucengdes.2011.07.042

177 Brkić, D. (2012). Determining friction factors in turbulent pipe flow. Chemical Engineering (New York),

178119 (3), 34-39. Available from: http://www.chemengonline.com/determining-friction-factors-in-

179 turbulent-pipe-flow/?printmode=1

180 Brkic, D. (2016). "Spreadsheet-based pipe networks analysis for teaching and learning purpose."

181 Spreadsheets in Education (eJSiE), 9(2), 4646. Available at: https://sie.scholasticahq.com/article/4646.pdf 182 Brkić, D. (2017a). "Solution of the implicit Colebrook equation for flow friction using Excel."

183 Spreadsheets in Education (eJSiE), 10(2). 4663. Available at:

184 https://sie.scholasticahq.com/article/4663.pdf

185 Brkić, D. (2017b). “Discussion of 'Exact analytical solutions of the Colebrook-White equation' by Yozo

186 Mikata and Walter S. Walczak.” Journal of Hydraulic Engineering, 143(9), 07017007.

187 https://doi.org/10.1061/(ASCE)HY.1943-7900.0001341

188 Brkić, D., and Ćojbašić, Ž. (2016). "Intelligent flow friction estimation.” Computational Intelligence and 189 Neuroscience, Article ID 5242596. http://dx.doi.org/10.1155/2016/5242596 
Brkić, D., and Ćojbašić, Ž. (2017). “Evolutionary optimization of Colebrook’s turbulent flow friction

191 approximations.” Fluids, 2(2), 15. https://doi.org/10.3390/fluids2020015

192 Brkić, D., and Praks, P. (2019). "Accurate and efficient explicit approximations of the Colebrook flow

193 friction equation based on the Wright $\omega$-function." Mathematics, 7(1), 34.

194 https://doi.org/10.3390/math7010034

195 Chen, N. H. (1979). “An explicit equation for friction factor in pipe.” Industrial \& Engineering Chemistry

196 Fundamentals, 18(3), 296-297. https://doi.org/10.1021/1160071a019

197 Clamond, D. (2009). "Efficient resolution of the Colebrook equation.” Industrial \& Engineering

198 Chemistry Research, 48(7), 3665-3671. https://doi.org/10.1021/ie801626g

199 Ćojbašić, Ž., and Brkić, D. (2013). "Very accurate explicit approximations for calculation of the

200 Colebrook friction factor.” International Journal of Mechanical Sciences, 67, 10-13.

201 https://doi.org/10.1016/j.ijmecsci.2012.11.017

202 Colebrook, C. and White, C. (1937). "Experiments with fluid friction in roughened pipes." Proceedings of

203 the Royal Society of London. Series A, Mathematical and Physical Sciences 161(906), 367-381.

204 https://dx.doi.org/10.1098/rspa.1937.0150

205 Colebrook, C. F. (1939). "Turbulent flow in pipes with particular reference to the transition region

206 between the smooth and rough pipe laws." Journal of the Institution of Civil Engineers (London) 11(4),

207 133-156. https://dx.doi.org/10.1680/ijoti.1939.13150

208 Dubčáková, R. (2011). “Eureqa: software review.” Genetic Programming and Evolvable Machines, 12(2),

209 173-178. https://doi.org/10.1007/s10710-010-9124-Z

210 Džunić, J., Petković, M. S., and Petković, L. D. (2011). “A family of optimal three-point methods for 211 solving nonlinear equations using two parametric functions.” Applied Mathematics and Computation, 212 217(19), 7612-7619. https://doi.org/10.1016/j.amc.2011.02.055

213 Eureqa [computer software]. Nutonian, Inc., Boston, MA. 
214 Giustolisi, O., Berardi, L., and Walski, T. M. (2011). "Some explicit formulations of Colebrook-White

215 friction factor considering accuracy vs. computational speed.” Journal of Hydroinformatics, 13(3), 401-

216 418. https://doi.org/10.2166/hydro.2010.098

217 Gregory, G. A., and Fogarasi, M. (1985). “Alternate to standard friction factor equation.” Oil and Gas

218 Journal, 83(13). 125-127.

219 MATLAB [computer software]. MathWorks, Inc., Natick, MA.

220 Mikata, Y., and Walczak, W. S. (2016). "Exact analytical solutions of the Colebrook-White equation."

221 Journal of Hydraulic Engineering, 142(2), 04015050. https://doi.org/10.1061/(ASCE)HY.1943-

$222 \quad 7900.0001074$

223 Özger, M., and Yıldırım, G. (2009). "Determining turbulent flow friction coefficient using adaptive

224 neuro-fuzzy computing technique.” Advances in Engineering Software, 40(4), 281-287.

225 https://doi.org/10.1016/j.advengsoft.2008.04.006

226 Praks, P., and Brkić.D. (2018a). "Symbolic regression-based genetic approximations of the Colebrook

227 equation for flow friction.” Water, 10(9), 1175. https://doi.org/10.3390/w10091175

228 Praks, P., and Brkić.D. (2018b). “Advanced iterative procedures for solving the implicit Colebrook

229 equation for fluid flow friction.” Advances in Civil Engineering, 2018, 5451034.

230 https://doi.org/10.1155/2018/5451034

231 Praks, P., and Brkić.D. (2018c). "Choosing the optimal multi-point iterative method for the Colebrook

232 flow friction equation.” Processes, 6(8), 130. https://doi.org/10.3390/pr6080130

233 Praks, P., and Brkić.D. (2018d). "One-log call iterative solution of the Colebrook equation for flow

234 friction based on Padé polynomials.” Energies, 11(7), 1825. https://doi.org/10.3390/en11071825

235 Schorle, B. J., Churchill, S. W., and Shacham, M. (1980). “Comments on: 'An explicit equation for

236 friction factor in pipe'.” Industrial \& Engineering Chemistry Fundamentals, 19(2), 228-230.

237 https://doi.org/10.1021/i160074a019

238 Schmidt, M. and Lipson, H. (2009). "Distilling free-form natural laws from experimental data.” Science 239 324(5923), 81-85. https://doi.org/10.1126/science.1165893 
240 Serghides, T.K. (1984). "Estimate friction factor accurately." Chemical Engineering (New York), 91(5),

241 63-64.

242 Sharma, J. R., and Arora, H. (2016). "A new family of optimal eighth order methods with dynamics for

243 nonlinear equations.” Applied Mathematics and Computation, 273, 924-933.

244 https://doi.org/10.1016/j.amc.2015.10.049

245 Sonnad, J. R., and Goudar, C. T. (2006). "Turbulent flow friction factor calculation using a

246 mathematically exact alternative to the Colebrook-White equation.” Journal of Hydraulic Engineering,

247 132(8), 863-867. https://doi.org/10.1061/(ASCE)0733-9429(2006)132:8(863)

248 Vatankhah, A. R. (2014). "Comment on 'Gene expression programming analysis of implicit Colebrook-

249 White equation in turbulent flow friction factor calculation'.” Journal of Petroleum Science and

250 Engineering, 124, 402-405. https://doi.org/10.1016/j.petrol.2013.12.001

251 Vatankhah, A. R., and Kouchakzadeh, S. (2008). "Discussion of 'Turbulent flow friction factor

252 calculation using a mathematically exact alternative to the Colebrook-White equation' by Jagadeesh R.

253 Sonnad and Chetan T. Goudar.” Journal of Hydraulic Engineering, 134(8), 1187-1187.

254 https://doi.org/10.1061/(ASCE)0733-9429(2008)134:8(1187)

255 Vatankhah, A. R., and Kouchakzadeh, S. (2009). "Discussion: Exact equations for pipe-flow problems."

256 Journal of Hydraulic Research 47(4), 537-538. https://doi.org/10.1080/00221686.2009.9522031

257 Winning, H. K., and Coole, T. (2013). "Explicit friction factor accuracy and computational efficiency for 258 turbulent flow in pipes.” Flow, Turbulence and Combustion, 90(1), 1-27. https://doi.org/10.1007/s10494$259 \quad 012-9419-7$

260 Zigrang, D. J. and Sylvester, N. D. (1982). "Explicit approximations to the solution of Colebrook's

261 friction factor equation.” AIChE Journal, 28(3), 514-515. https://dx.doi.org/10.1002/aic.690280323 